\title{
GARRAFAS PET COMO ALTERNATIVA PARA A CONFECÇÃO DE RECIPIENTES PARA CRIAÇÃO DE INSE- TOS EM LABORATÓRIO
}

\author{
Iracilda Maria de Moura Lima ${ }^{1 *}$; Maria Bernadete de Carvalho ${ }^{1}$
}

\begin{abstract}
${ }^{1}$ Universidade Federal de Alagoas, Instituto de Ciências Biológicas e da Saúde (ICBS). Av. Lourival Melo Mota, s/n, Tabuleiro do Martins - Maceió-AL, CEP: $57072-970$

*Autor para correspondência: Iracilda Maria de Moura Lima, iracilda.lima@icbs.ufal.br
\end{abstract}

\begin{abstract}
RESUMO: Visando minimizar os custos da pesquisa e o problema operacional de manipulação diária, a presente comunicação sugere diversos recipientes para a criação de insetos construídos a partir de garrafas descartáveis confeccionadas com tereftalato de polietileno (PET). A partir do princípio do encaixe do terço superior da garrafa como cúpula numa base larga de tampa de alimentos lácticos com $15 \mathrm{~cm}$ de diâmetro, propõem-se um recipiente de criação. Esses recipientes têm se revelado muito eficazes por garantirem um ambiente adequado para a criação de insetos com diminuição do stress e da possibilidade de fuga; praticidade no manejo; possibilidade de utilização em grande quantidade por ser material descartado, garantindo baixo custo.
\end{abstract}

PALAVRAS-CHAVE: Experimentação, Desenvolvimento, Parasitoidismo, Educação Ambiental.

ABSTRACT: Alternative containers for rearing insects in laboratory setting - Several insect rearing cages are suggested herein, in order to keep to a minimum both research costs and operacional problems of insect daily manipulation. These enclosures are made of polyethylene terephthalate (PET)soda bottles embedded in 15-cm diameter plastic lids for dairy products in which a dome-shaped top fits onto a shallow base. Since in most containers access is from the top,the ease and safety during the cleaning process is the differential of this cage proposed here. The base can thus, be easily replaced during cleaning, hindering escape and causing no undue distress to the insects inside. These features lead toeffectiveness which is perceived by the quality of the rearing environment that reduces stress; to convenience in handling; as well as the possibility of use in large quantities of discarded material, ensuring low cost.

KEYWORDS: Experimentation, Development, Parasitoidism, Environmental Education.

Estudos sobre desenvolvimento, comportamento e fisiologia de insetos em condições de laboratório, além de fornecerem indicadores que apoiam a busca por medidas mais adequadas para controle de pragas, também tem contribuído para o desenvolvimento de técnicas de criação de insetos em laboratório (Quesada-Moraga e Santiago-Álvarez, 2001), incluindo a proposta de vários tipos de recipientes para criação.

A falta de destaque dos conteúdos da metodologia quando comparados aos apresentados como resultado torna baixa a visibilidade de informações inéditas. Assim, a forma como tradicionalmente os recipientes de criação são descritos nos artigos científicos, pode diminuir a possibilidade de seu uso, e, consequentemente, de sua citação. Tentando realçar a importância das técnicas de criação, muitos periódicos permitem sua publicação na forma de comunicação científica, como estratégia de destacá-las, aumentan- do a possibilidade de sua seleção. Como exemplo de aumento da visibilidade, tem-se o protocolo e técnica de criação massal de Leucoptera coffeellum (Guérin-Mèneville \& Perrottet, 1842) (Lepidoptera: Lyonetiidae) (Reis-Júnior et al., 2000); a proposta de "unidade de criação simples" para o inseto hospedeiro e seu parasitoide Cotesia platellae (Kurdjumov, 1912) (Lim \& Chan, 1986); técnica de criação para Mahanarva fimbriolata (Stål, 1854) (Hemiptera: Cercopidae) (Garcia et al., 2007); para Halymorpha halys Stål, 1855 (Heteroptera: Pentatomidae) (Medal et al., 2012);

A preocupação com a publicação da descrição de opções para a contenção de insetos não é recente, sendo localizados trabalhos datados do início e de meados do século passado como o de Hartley (1923), para a criação de Aphelinussemi flavus (Howard, 1908) (Hymenoptera: Aphelinidae); o de Horsburgh e Asquith (1969) com a proposta de recipiente de criação para 
estudos em laboratório com Miridae e Anthocoridae (Hemiptera: Heteroptera). Essa importância tem sido reconhecida por instituições como o United States Department of Agriculture (USDA) que chegou a editar um livro contendo artigos de revisão sobre todos os cuidados para a otimização da criação de insetos em laboratórios e em insetários (King e Leppla, 1984).

Essa postura também tem sido adotada por entidades científicas como a Entomological Societyof Ontario, no Canadá, que divulgam formas simples de construção de gaiolas para criação de insetos (Corrigan, 2004), atraindo e aumentando o número de admiradores desse grupo de animais. Universidades americanas, como a Michigan State University, apresentam programas de extensão com iniciativas semelhantes como a edição de manuais para estimular estudantes a observar o comportamento dos insetos a partir da criação (Dunn, 1989).

Além da contenção dos espécimes em grupo ou isolados, um recipiente de criação deve apresentar algumas características que the garantam praticidade no manejo (principalmente em relação à limpeza, garantindo um ambiente salubre, com um mínimo de contaminação por micro-organismos) sem que haja comprometimento da segurança em relação à contenção dos insetos, permitindo, ainda, facilidade de visualização (O'brien, 1984) para o acompanhamento de fenômenos biológicos relacionados a aspectos como: (1) uso do tempo durante a vida dos insetos fitófagos; (2) determinação da taxa de desenvolvimento; (3) determinação da razão sexual; (4) dinâmica da reprodução incluindo produção de ovos; (5) estudo de interações inter-específicas, como o parasitoidismo; e (6) descrição do comportamento, mobilidade, entre outros (Leppla, 2008).

Além dos recipientes anteriormente indicados e que foram confeccionados a partir de adaptações de materiais disponíveis, vários são produzidos por empresas especializadas (Megaview, 2007; Bioquip, 2015; Educational Science, 2015), cujo preço pode variar, de centavos a centenas de dólares. No entanto, muitos desses recipientes comercializados, além de relativamente caros, também não são práticos, por suas características indesejáveis principalmente em relação à limpeza e visualização dos eventos biológicos.

Considerando-se que a demanda de recipientes para o desenvolvimento de um trabalho científico geralmente é alta — dezenas ou mesmo centenas-a realização de experimentos pode até ser inviabilizada em função dos altos custos. Esse tem sido um dos principais motivos para se investir na improvisação de uma infinidade de containers, muitos deles com custo de produção próximo de zero, como no caso do aproveitamento do bojo de vidro de lâmpadas incandescentes para oviposição de Helicoverpa armigera (Hübner, 1805) (Ahmed et al., 1998; Abbasi et al., 2007) e outras propostas para criação de insetos de várias espécies e hábitos (Dunn, 1989).

A grande maioria dos trabalhos publicados registra, na metodologia, recipientes improvisados, cuja estrutura predominante consiste numa parte inferior de vidro ou de plástico transparente, com abertura superior coberta com tecido, geralmente organdi de náilon, mantido preso por liga de borracha ou por elástico. Esse cuidado é tomado como forma de garantir a aeração do interior do recipiente e impedir a penetração de inimigos naturais, principalmente parasitoides. Muitas vezes, a própria tampa de frascos depois de perfuradas também são utilizadas.

No caso dos recipientes que apresentam tal princípio de funcionamento, deve-se ter muita atenção para que os insetos não fujam nem sejam feridos durante 0 manuseio diário. Durante a realização da limpeza e troca de alimento, geralmente é substituída a parte inferior, com transferência do inseto, o que pode provocar mais estresse ao inseto.

Visando minimizar a questão dos custos da pesquisa e o problema técnico de manipulação diária, a presente comunicação sugere diversas gaiolas para a criação de insetos a partir de garrafas de refrigerantes confeccionadas com tereftalato de polietileno (PET) e tampas plásticas de alimentos láticos, adotando como princípio de construção um sistema onde uma cúpula se acopla a uma base rasa.

Para a confecção dessas gaiolas foram providenciadas garrafas de refrigerante, de preferência da marca CocaCola $\AA$, com volume de $2 \mathrm{~L}$, tendo-se o cuidado de se retirar a tampa e o respectivo anel basal. Com 0 auxílio de caneta Pentel囚 especial para escrita em vidro foram feitas marcações segundo as indicações da Figura 1. Em seguida, com uma faca de serra ou de uma faca Olfaß, fazer uma incisão inicial em cada uma das marcações e, com o auxílio de uma tesoura média desmembrar em três partes. Às vezes, torna-se necessário fazerem-se pequenas aparas na base da cúpula para que se encaixe perfeitamente na tampa. 
Figura 1. Pontos de corte em uma garrafa Pet com capacidade de $2 \mathrm{~L}$ para construção de dois tipos de recipientes de criação

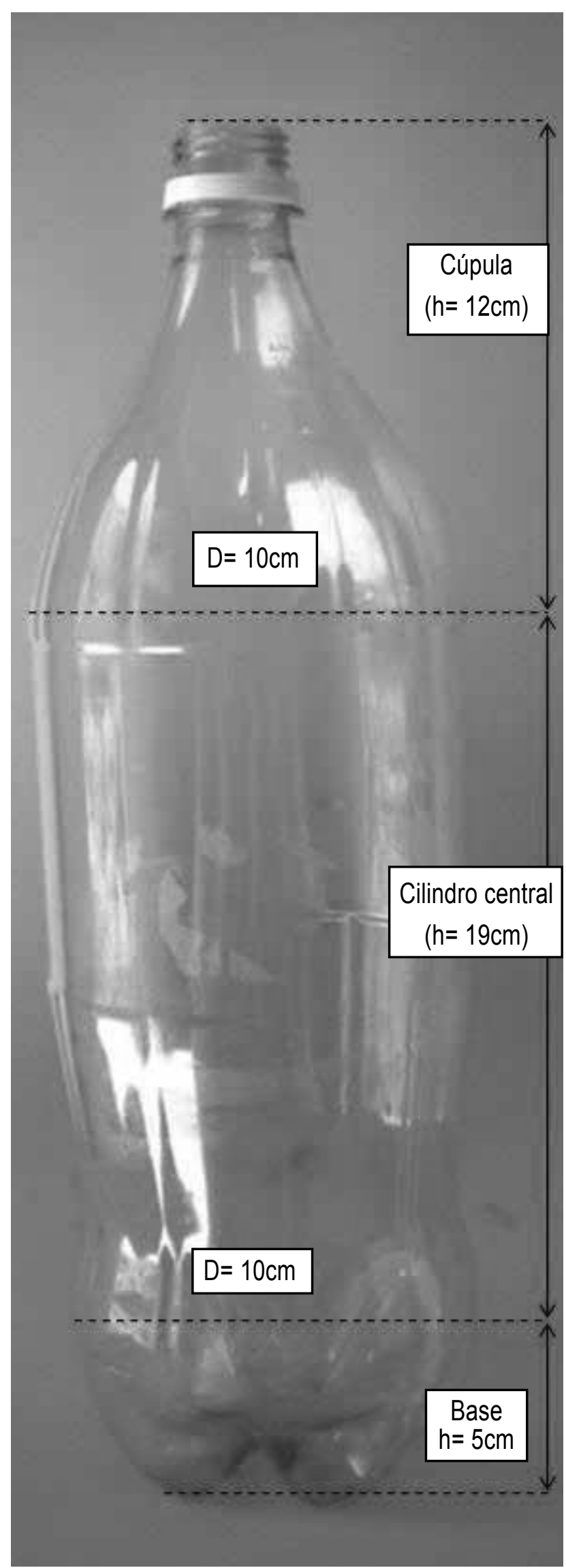

A garrafa pode ser dividida de duas formas. No primeiro caso, divide-se em três partes (Fig. 1): a superior ou cúpula (volume interno com cerca de 300 $\mathrm{mL}$ ), que será utilizada para a confecção da gaiola-padrão (Fig. 2A); a central, um cilindro (volume interno com cerca de 1,5 L) utilizado para compor a gaiola maior (Fig. 2B); e a base, que é descartada. No segundo caso, divide-se em duas partes, cortando-se apenas na região da base da garrafa (Fig. 1), obtendo-se a gaiola indicada na Figura $2 \mathrm{C}$, com quase 1,8 $L$ de volume interno.

Em todos os casos, a base das cúpulas obtidas após os cortes (base das cúpulas e parte inferior do cilindro) deve ter diâmetro de $10 \mathrm{~cm}$, para que possam se encaixar perfeitamente na base - tampas de leite em pó da marca Nestlé ${ }^{\circledR}$ (Fig. 2A, 2B e 2C).

$\mathrm{Na}$ abertura superior da garrafa deve ser colocado um pedaço de organdi de náilon em forma de quadrado com cerca de $6 \mathrm{~cm}$ de lado. Para evitar que o tecido desfie, recomenda-se aproximar as bordas, cuidadosamente, da chama de uma vela (Fig. 2A, 2B e 2C).

Como o protótipo foi proposto pela segunda autora deste trabalho, os estagiários e bolsistas do laboratório passaram a denominá-la de "gaiola-bernadete". Nessa versão o organdi ainda era fixado com liga de borracha, garantindo a circulação de ar, evitando que o inseto isolado no interior sofra a ação de inimigos naturais.

Atualmente a fixação tem sido feita com o anel colorido previamente removido da garrafa (Fig. $2 \mathrm{~A}, 2 \mathrm{C}$ e $2 \mathrm{D}$ ), o que é recomendado, uma vez que a variação de cores permite que facilmente sejam identificados grupos de recipientes, por exemplo, insetos que estejam submetidos a um mesmo tratamento estatístico; ou que se encontrem no mesmo estágio de desenvolvimento. 
Figura 2. Recipientes para criação de insetos construídos com garrafas Pet. A - Gaiola-bernadete contendo pupa e exúviade larva de quinto ínstar de Danausplexippuserippus (Cramer, [1775]) (Lepidoptera: Nymphalidae: Danainae); B - Gaiola cilíndrica; C - Adaptação para insetos maiores; D - Gaiola-bernadete para acompanhamento de insetos em plantas envasadas.
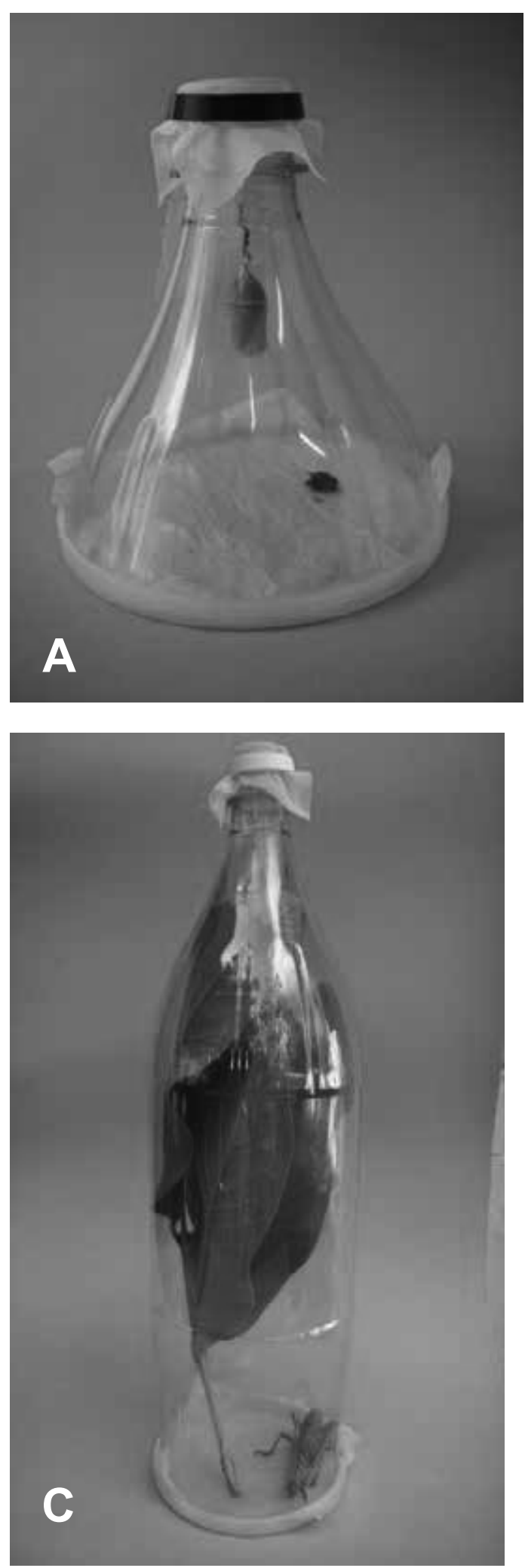

O surgimento no mercado das garrafas PET de $3 \mathrm{~L}$ permitiu com que fossem feitas gaiolas com volume maior utilizando como base a tampa da lata de $500 \mathrm{~mL}$ do produto Neston da marca Nestlé.
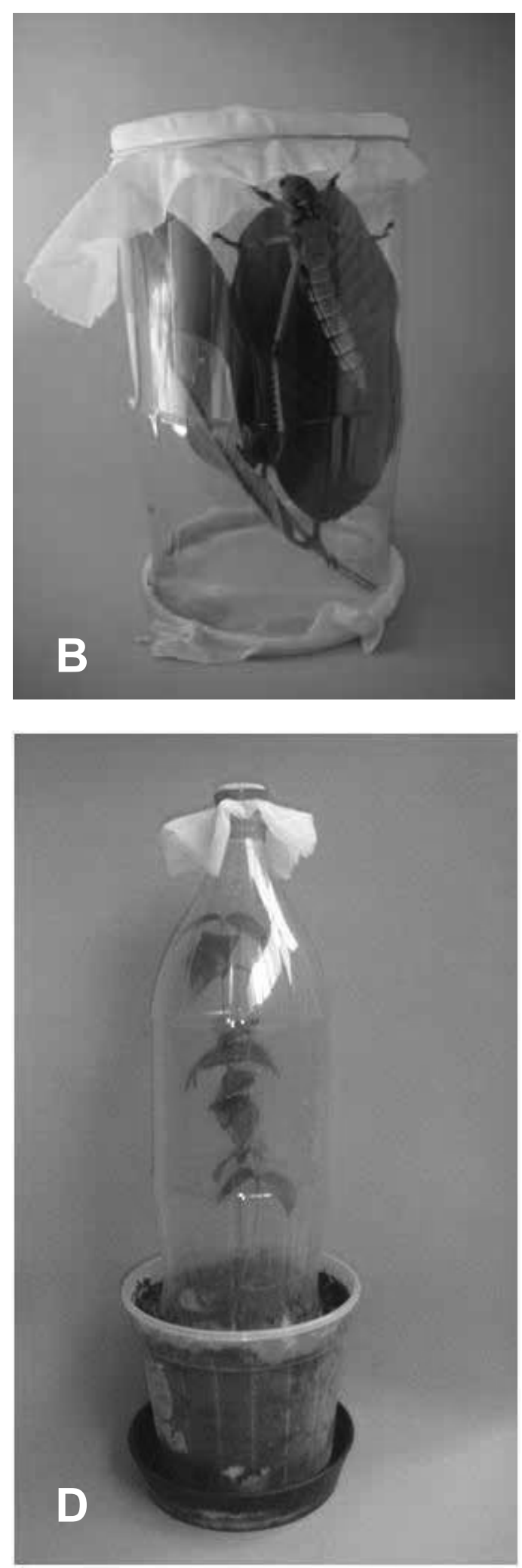

A praticidade dos recipientes que utilizam esse sistema pode ser verificada pelas vantagens a seguir enumeradas: (1) volume interno compatível com a maioria dos insetos disponíveis para observação; (2) 
fácil limpeza pela lavagem com sabão (Townsend e Bessin, 2004) e desinfestação com solução de hipoclorito de sódio a $5 \%$, conforme recomendações metodológicas feitas por Hirose e Neves (2002); (3) possibilidade de secagem ao sol (Townsend e Bessin, 2004), 0 que contribui para a eliminação de micro-organismos; (4) exigência de pouco espaço para armazenamento, pois permitem a sobreposição; (5) possibilidade de deslocamentos no interior do laboratório com facilidade e segurança - desde que a cúpula esteja bem encaixada na base, é suficiente segurá-la pelo gargalo; (6) facilidade de troca de alimento minimizando o estresse do espécime que está sendo estudado e, ainda, diminuindo as possibilidades de fuga do espécime (no caso de saltadores ou de adultos alados), uma vez que a cúpula pode ser removida com um mínimo de movimento; (7) longo tempo útil de utilização, pois podem ser reutilizadas por tempo indeterminado; e (8) facilidade de armazenamento das cúpulas encaixadas.

Além de todas essas vantagens dos recipientes aqui propostos, trabalhos já realizados desde 1999 no Laboratório de Bioecologia de Insetos do Instituto de Ciências Biológicas e da Saúde da Universidade Federal de Alagoas (por exemplo: Barros e Lima, 2004; Born e Lima, 2005; Andrade, 2005; Marcicano et al., 2007; Santos, 2007; Dantas-Júnior, 2009) têm demonstrado que estudos sobre o desenvolvimento de lepidópteros podem ser realizados com facilidade: garantem o espaço necessário para o desenvolvimento das larvas, fixação das pupas e distensão das asas, sem comprometer a obtenção de adultos viáveis e em bom estado para montagem e incorporação a coleções entomológicas; ou liberação no ambiente.

Essas vantagens superam aquelas propostas por Hirose e Neves (2002) ao discutirem sua técnica de criação de insetos em laboratório: (1) fácil manutenção; (2) possibilidade de controle de contaminações, (3) baixa demanda de tempo de manipulação, (4) possibilidade de rápida ampliação no caso de necessidade de maior número de insetos; (5) impedimento do contato dos insetos com substâncias que podem alterar os resultados dos bioensaios; e (6) minimização do estresse pelo manuseio.

Esses recipientes têm sido utilizados para criação principalmente de lepidópteros, ortópteros, himenópteros e dípteros. Também são indicados para o desenvolvimento de estudos para a determinação do parasitoidismo em pupas de lepidópteros (Marci- cano et al., 2007, 2009), desde que se coloquem três camadas de organdi, para evitar que os parasitoides como os dos gêneros Brachymeria e Conura (Hymenoptera: Chalcididae) façam orifícios para fuga (Marcicano et al., 2007).

A gaiola cilíndrica e a gaiola de 1,8 L, apesar de menos práticas, são indicadas para a criação de insetos maiores (Fig. 2B e $2 \mathrm{C}$ ), e apresentam quase todas as vantagens da anterior. No entanto, exigem um maior espaço para armazenamento em armários (não podem ser encaixadas). No caso da gaiola cilíndrica (Fig. 2C), o seu deslocamento no laboratório é menos seguro, pois o organdi fixado com liga de borracha na parte superior pode se desprender com mais facilidade, assim como o encaixe na parte basal. Por isso, quando for necessária a transferência de local, deve-se segurá-la pela base: uma mão deve ser mantida sob a tampa plástica da lata de leite em pó, enquanto a outra se apoia na parte externa do cilindro.

A cúpula também se presta para isolar insetos quando observados em plantas envasadas (Fig. 2D). Nesse caso deve-se utilizar toda a garrafa cortando-se apenas a base para que seja introduzida no solo, impedindo a fuga dos insetos. Ao se utilizar as gaiolas em vasos, prepara-se a cúpula, cuidando para que a base se encaixe perfeitamente no diâmetro da base do vaso. Nesse caso, a linha de corte não precisa ser tão precisa. Essas cúpulas podem até ser utilizadas no campo sobre plantas, sendo inseridas diretamente no solo.

0 diferencial dos recipientes propostos é o princípio utilizado: enquanto na grande maioria dos recipientes o acesso é pela parte superior - 0 que pode facilitar a fuga do inseto - nas versões aqui apresentadas a abertura maior na parte inferior permite que a base seja substituída facilmente durante o processo de limpeza, dificulta a fuga e provoca um mínimo de stress no inseto que permanece em seu interior.

Além da utilidade para fins científicos, por serem resultado do reuso de materiais recicláveis, essas gaiolas também se prestam para experiências em ciências no ensino fundamental, de modo a desenvolver nos estudantes diversas habilidades e atitudes positivas em relação à vida e ao meio ambiente (Educação Ambiental). Nesse caso em particular, entre os objetivos educacionais esperados destacam-se: 0 aprendizado sobre o ciclo de vida dos insetos; o estímulo do aprendizado cooperativo; o desenvolvimento da responsabilidade a partir do cuidado com os animais; 
habilidade de comunicação oral e escrita, a partir das descrições dos fenômenos observados, da comparação dos insetos com outros animais, da descoberta do papel dos insetos no meio ambiente, entre outros (Smithsonian Institution, 2006).

Como atualmente, a diversidade de recipientes e das cores das tampas tanto das garrafas PET como as das latas dos produtos lácteos (branca, azul, vermeIha, amarela, preta entre outras), as combinações entre essas cores podem ser utilizadas como código, para que o técnico de laboratório visualize melhor e identifique facilmente situações experimentais (por exemplo: diferentes tratamentos aplicados; estágio de desenvolvimento do/s inseto/s; ou tipo de fenômeno que está sendo estudado).

Torna-se importante salientar, que, embora esteja estabelecido que algumas cores - como 0 amarelo - possam interferir no comportamento de insetos, nesse caso isso não ocorre: a base (tampa invertida de lata de leite em pó) fica revestida por papel absorvente (guardanapo de papel, papel-toalha, ou papel-filtro).

Considerando-se a grande diversidade de tampas e de garrafas atualmente disponíveis no mercado, passam a ser inúmeras as variações em torno desse mesmo tema: podem-se utilizar garrafas menores e tampas plásticas de menor diâmetro, ou garrafas maiores, como as de $3 \mathrm{~L}$ e bases maiores, como a tampa do produto Neston $₫$ da marca Nestlé ${ }^{\circledR}$, permitindo que o recipiente tenha cerca de 500 $\mathrm{mL}$ de volume interno.

Esses recipientes têm se revelado muito eficazes tanto por garantirem um ambiente adequado para a criação de insetos; pela praticidade no manejo de troca de alimento com substituição da base; possibilidade de serem utilizados em grande quantidade; e pela facilidade de visualização dos eventos biológicos (comportamento alimentar, acasalamentos, oviposição, eclosão, ecdises, emergência, morte, saída de parasitoides do hospedeiro) que ocorrem no interior do recipiente.

\section{AGRADECIMENTOS}

A Gilberto Costa Justino, do Setor de Botânica do Instituto de Ciências Biológicas e da Saúde, pelas sugestões e revisão do texto.

\section{REFERÊNCIAS BIBLIOGRÁFICAS}

Abbasi, B.H.; Ahmed, K.; Khalique, F.; Ayub, N.; Liu, H.J.; Kazmi, S.A.R.; Aftab, M.N. Rearing the cotton bollworm, Helicoverpa armigera, on a tapioca-based artificial diet. Journal of Insect Science. 2007, 7, 35, 7p. Disponível em: <http://www.insectscience.org/7.35/ i1536-2442-2007-35.pdf>. Acesso: 6 jul 2007.

Andrade, L.H. Indicadores do desenvolvimento ontogenético de Stiphrasp. x (Orthoptera: Proscopiidae) alimentando-se de folhas de PsidiumguajavaL. (Myrtaceae) em laboratório. Rio Largo: CECA-UFAL. Originalmente apresentada como dissertação de Mestrado, Universidade Federal de Alagoas. 2005.

Barros, W.R.S.; Lima, I.M.M.. Desenvolvimento pré-imaginal de Eueidesisabelladianasa (Hübner) (Lepidoptera, Nymphalidae, Heliconiinae) em folhas de Passiflora edulis L. (Passifloraceae). Revista Brasileira de Entomologia, 2004, 48, 1, 69-75.

Bioquip. Bioquip products since 1947: Equipment, Supplies and Books For Entomology and Related Sciences. Rancho Domingues: BIOQUIP. 2015. Disponível em: <https://www. bioquip.com/>. Acesso: 26 ago 2015.

Born, F.S.; Lima, I.M.M. Desenvolvimento pós-embrionário de Anteosmenippe (Hübner) (Lepidoptera, Pieridae) em Cassia ferruginea Shrad. (Caesalpinaceae), em laboratório. Revista Brasileira de Entomologia, 2005, 49, 4, 522-526.

Corrigan, J. Home made entomology equipment: plastic insect cages. ESO Newsletter, 2004, 9, 2, 12. Disponivel em: <http://www.entsocont.cal uploads/3/0/2/6/30266933/v_9_issue_2_summer_2004.pdf>. Acesso em: 14 dez 2016.

Dantas Júnior, A.M.M. Desenvolvimento de Neomegaloto musparvus (Westwood, 1842) (Hemiptera: Alydidae) em planta alimentícia alternativa, Macroptilium atropurpureum Urb. (Leguminosae: Papilionoideae), em laboratório. CECA-UFAL. Originalmente apresentada como dissertação de mestrado, Universidade Federal de Alagoas, 48p. 2009.

Dunn, G.A. Insect life cycle studies. East Lansing: Michigan State University Extension. 1989. 37p. (4H 1406 En- 
tomology Manual, 3). Disponível em: <http://entnemdept. ufl.edu/bug_club/teacher-parents/pdf/4H1406InsectLifeC ycleStudiesManual3.pdf>. Acesso em: 26 ago 2015.

EDUCATIONAL SCIENCE. Online science and nature store: Insect Rearing Supplies and Equipment. League City: Educational Science. 2015. Disponível em: $<$ http://www.educationalscience.com/>. Acesso em: 26 ago 2015.

Garcia, J.F.; Botelho, P.S.M.; Parra, J.R.P. Laboratory rearing technique of Mahanarva fimbriolata (Stål) (Hemiptera: Cercopidae). Scientia Agricola, 2007, 64, 1, 73-76. Disponível em: < http://www.scielo.br/pdf/sa/ v64n1/a11v64n1.pdf>. Acesso em: 14 dez 2016.

Gyeltshen, J.; Bernon, G.; Hodges, A. Brown Marmorated Stink Bug, Halyomorpha halys Stål (Insecta: Hemiptera: Pentatomidae). Tallahassee: University of Florida Institute of Food and Agricultural Sciences Extension. 2013. 7p. (Featured Creatures from the Entomology and Nematology Department - Florida Cooperative Extension Service - IFAS, EENY-346).

Hartley, E.A.. Some bionomics of Aphelinus semiflavus (Howard), chalcid parasite of aphids. Ohio Journal of Sciences, 1923, 22, 209-236.

Hirose, E.; Neves, P.M.O.J. Técnica para Criação e Manutenção da Broca-do-Café, Hypothenemu shampei (Ferrari) (Coleoptera: Scolytidae), em Laboratório. Neotropical Entomology, 2002, 31, 1, 161-164.

Horsburgh, R.L.; Asquith, D. Laboratory rearing cage suitable for studies of predaceous mirids and anthocorids collected on apple. Journal of Economic Entomology, 1969, 62, 5, 1242. Disponível em: <http://jee. oxfordjournals.org/content/62/5/1242.1 >. Acesso em: 14 dez 2016.

Leppla, N.C. Rearing of Insects. P. 3101-3108. In CAPINERA, J.L. (Ed.), Encyclopedia of Entomology, 2. Ed. Dordrecht: Springer. 2008. Disponível em: <http://link.springer.com/referenceworkentry/10.1007\% 2F978-1-4020-6359-6_3306>. Acesso em: 03 jun 2015.

Lim, G.S.; Chan, H.F. A Simple rearing unit for diamond back moth and its parasitoid Apanteles platellae Kurdj. Mardi Research Bulletin, 1986, 14, 2, 154-157.
King, E.G.; Leppla, N.C. (eds.). Advances and challenges in insect rearing. New Orleans: Agricultural Research Service-USDA. 1984. 306p.

Marcicano, M.D.L.; Nihei, S.S.; Lima, I.M.M. First Host Record for Winthemia analis (Macquart) (Diptera: Tachinidae: Exoristinae) in Brazil: Brassolis sophorae laurentii Stichel (Lepidoptera: Nymphalidae: Brassolinae). Neotropical Entomology, 2009, 38, 4, 550-552.

Marcicano, M.D.L.; Lima, I.M.M.; Tavares, M.T.; Casagrande, M.M.. Parasitism of Brassolis sophorae laurentii Stichel (Lepidoptera: Nymphalidae, Brassolinae) Pupae by Conura morleyi (Ashmead) (Hymenoptera: Chalcididae, Chalcidini), in the State of Alagoas, Brazil. Neotropical Entomology, 2007, 36, 4, 629-631.

Medal, J.; Smith, T.; Fox, A.; Santa-Cruz, A.; Poplin, A.; Hodges, A. Rearing the brown marmorated stink bug Halyomorpha halys (Heteroptera: Pentatomidae). Florida Entomologist, 2012, 95, 3, 800-802.

MEGAVIEW. Bugdorm store. 2007. Disponível em: <http:// educationalscience.com/insectrearing.htm>e <http://bugdorm.megaview.com.tw/>. Acesso em: 05 jul 2007.

O'brien, M.F. Rearing Insects Indoors. East Lansing: Michigan Entomological Society/Michigan State University. (Entomology Notes, 15). 1984. Disponível em: $<$ http://insects.ummz.Isa.umich.edu/MES/notes/entnotes15.html>. Acesso em: 05 jul 2007.

Quesada-Moraga, E.; Santiago-Álvarez, C. Rearing and breeding of the Moroccan locust Dociostaurus maroccanus (Thunberg) (Orthop., Acrididae) under laboratory conditions. Journal of Applied Entomology, 2001, $125,3,121-124$.

Reis-Junior, R.; Lima, E.R.; Vilela, E.F.; Barros, R.S. Method for Maintenance of Coffee Leaves In Vitro for Mass Rearing of Leucoptera coffeellum (Guérin-Méneville) (Lepidoptera: Lyonetiidae). Anais da Sociedade Entomológica do Brasil, 2000, 29, 4, 849-854.

Santos, I.P. Aspectos bioecológicos da associação de Dirphia sp. (Lepidoptera: Saturniidae: Hemileucinae) a Schinustere binthifolia Raddi (Anacardiaceae) (aroeira-da-praia), no Estado de Alagoas. Maceió: ICBS. 2007. Originalmente apresentada como Trabalho de Conclu- 
são de curso de graduação, Universidade Federal de Townsend, L.; Bessin, R. Rearing insects. CooperatiAlagoas. 59p. ve Extension Service-University of Kentucky-College of Agriculture. (Entfact, 007). 2004. Disponível em: < SMITHSONIAN INSTITUTION. Bugs: teacher's guide. http://www.ca.uky.edu/entomology/entfacts/ef007.asp Washington: Smithsonian Institution. 2006. 7p.

>. Acesso em: 25 mai 2009. 